\title{
The Emerging Clinical Utility of Neurosonology During COVID-19 Pandemic
}

Eleni Bakola, MD* (D); Odysseas Kargiotis, $\mathrm{MD}^{\dagger}$ (D); Klearchos Psychogios, MD ${ }^{*} \dagger$ (D); Apostolos Safouris, MD ${ }^{*} \dagger$ (D); Lina Palaiodimou, MD* (D); Maria-Ioanna Stefanou, MD* (D); Maria Chondrogianni, MD* (D);

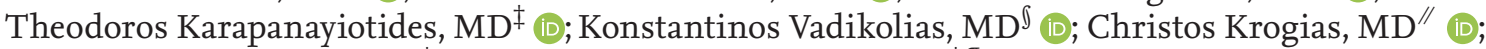

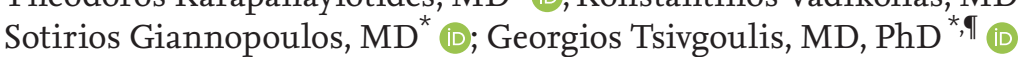

Second Department of Neurology, National \& Kapodistrian University of Athens, School of Medicine, "Attikon" University Hospital", Athens, Greece; Stroke Unit, Metropolitan Hospital ${ }^{\dagger}$, Piraeus, Greece; Second Department of Neurology, Aristotle University of Thessaloniki, School of Medicine, Faculty of Health Sciences, AHEPA University Hospital ${ }^{\ddagger}$, Thessaloniki, Greece; Department of Neurology, School of Medicine, Democritus University of Thrace ${ }^{\complement}$, Alexandroupolis, Greece; Department of Neurology, St. Josef-Hospital, Ruhr University Bochum //, Bochum, Germany; Department of Neurology, The University of Tennessee Health Science Center, Memphis ${ }^{9}$, Tennessee, USA

Coronavirus disease 2019 (COVID-19) is caused by severe acute respiratory syndrome coronavirus 2 infection. Over the last 2 years the virus has spread worldwide with enormous implications on the healthcare systems. COVID-19 patients suffer from mild upper-airway manifestations to pneumonia and severe acute respiratory distress syndrome and their hospitalizations are often prolonged. Neurological manifestations of the disease are common. Neurosonology (transcranial Doppler \& cervical duplex ultrasound) is an easily repeatable diagnostic imaging modality that can be simply applied at the bedside of COVID-19 patients with cerebrovascular diseases or in critically ill patients in the intensive care unit. Neurosonology may provide hemodynamic assessment of cerebral circulation, quantitative evaluation of increased intracranial pressure and detection of micro-embolic signals in real-time. Consequently, it may assist substantially in the diagnosis, risk stratification and therapeutic approach of COVID-19 patients with or without cerebrovascular complications. In the present narrative review, we discuss the emerging clinical utility of neurosonology during COVID-19 pandemic and highlight the upgraded role of neurosonology resulting from the combination of the established applications coupled with the reduced risk of virus spreading during ultrasound evaluation compared to other imaging modalities including computed tomography and magnetic resonance imaging.

J Neurosonol Neuroimag 2021;13(2):27-36

Key Words: SARS-CoV-2; Ultrasonography; Doppler; Transcranial; Intensive care unit
Received: August 28, 2021

Revised: October 18, 2021

Accepted: October 18, 2021

Correspondence:

Georgios Tsivgoulis, MD, $\mathrm{PhD}$

Second Department of Neurology, "Attikon" University Hospital, National and Kapodistrian University of Athens, School of Medicine, Rimini 1, Chaidari, Athens 12462, Greece Tel: $+30-6937178635$ Fax: $+30-2105832471$ E-mail: tsivgoulisgiorg@ yahoo.gr

\section{INTRODUCTION}

During the last 2 years coronavirus disease 2019 (COVID-19) pandemic stormed into the healthcare systems worldwide with over 200 million confirmed cases of severe acute respiratory syndrome coronavirus type 2 (SARS-CoV-2) infection and over 4 million deaths worldwide. ${ }^{1}$ Previous hypotheses that COVID-19 was not confined to the respiratory system, but it was also responsible for a syndrome of multiorgan dysfunction, including neurological involvement have been proved by several studies ${ }^{2}$ and nowadays COVID-19 is recognized as a multisystem disease with apparent involvement of other organ systems. ${ }^{3,4}$ Neurological manifestations vary from acute cerebrovascular events to immuno-mediated diseases. ${ }^{5}$

Cerebrovascular complications associated with COVID-19 include ischemic strokes, intracranial hem- 
orrhage and cerebral venous thrombosis. ${ }^{6}$ In particular, according to the meta-analysis by Katsanos et al. ${ }^{7}$, patients infected by SARS-CoV-2 appear to have increased odds of ischemic stroke rate when compared to contemporary or historical non-infected controls. Remarkable is also a 5-fold increased mortality risk for patients infected by SARS-CoV-2 suffering from cerebrovascular events compared to non-infected stroke patients.?

Healthcare systems are overwhelmed by the increased volume of admissions during the COVID-19 pandemic. In times of limited hospital resources and increased risk of infection spread to hospital personnel every useful pathway and diagnostic tool which is easily applicable at the bedside becomes valuable. ${ }^{8}$ The European Society of Neurosonology and Cerebral Hemodynamics recognizing the crucial role of neurosonology in the setting of COVID-19 pandemic released in 2020 practice recommendations for neurovascular ultrasound investigations of acute stroke patients. ${ }^{9}$ Neurosonology can be also applied in COVID-19 patients with other pathologies than stroke including increased intracranial pressure (ICP) and cerebral circulatory arrest. In this narrative review, we present the emerging neurosonology applications for COVID-19 patients and summarize the available literature that is relevant to this topic (Table 1 ).

\section{ULTRASOUND ASSESSMENT OF CERVICAL AND CEREBRAL VESSEL PATENCY IN COVID-19 PATIENTS WITH ACUTE STROKE}

All stroke patients should undergo a fast track COVID-19 nasopharyngeal test in order to prevent in-hospital transmission according to the recommendations of European Society of Neurosonology and Cerebral Hemodynamics. All sonographers should wear enhanced personal protective equipment (PPE) at all times: respirators (N95 or FFP2 masks), surgical cap, goggles, face shield, full-sleeved gown, two pairs (internal and external) of extended cuff gloves and outer short cuff gloves, boot-type shoe covers, and proper donning/doffing hygiene. In case of resource limitations, a standard surgical mask can be worn over the N95 or FFP2 mask for preservation of the latter. ${ }^{9}$

In case of COVID-19 positive and presumed positive patients presenting with acute stroke symptoms therapeutic decisions on systemic or endovascular reperfusion therapies may rely on ultrasound to avoid potential time delays related to CT or MR angiography. ${ }^{10}$ In addition, transcranial Doppler (TCD) can provide rapid information about vascular stenosis and occlusion, the hemodynamic status of the cerebral circulation, and real-time monitoring of recanalization. ${ }^{11,12}$ According to

TABLE 1. Neurosonology applications during COVID-19 pandemic

\begin{tabular}{|c|c|c|c|}
\hline Application & TCD & TCCD & Clinical relevance \\
\hline Screening & Yes & Yes & Avoid using CTA for LVO in COVID-19 patients with stroke ${ }^{9}$ \\
\hline Midline shift evaluation & No & Yes & Avoid using CT to evaluate cerebral edema in COVID-19 patients with stroke ${ }^{40}$ \\
\hline Screening for RLS & Yes & No & $\begin{array}{l}\text { - Avoid TEE in COVID-19 patients with stroke (in particular in negative TCD cases } \\
\text { for RLS) } \\
\text { - Detection of pulmonary vascular dilatation in patients with COVID-19 pneumo- } \\
\text { nia }{ }^{46}\end{array}$ \\
\hline Microemboli detection & Yes & No & $\begin{array}{l}\text { - Risk stratification of stroke recurrence risk in COVID-19 stroke patients }{ }^{11} \\
\text { - Monitoring the response to anticoagulation in COVID-19 patients with stroke }\end{array}$ \\
\hline Vasomotor reactivity & Yes & No & Assessment of cerebral hemodynamic reserve in COVID-19 patients ${ }^{22,25}$ \\
\hline $\begin{array}{l}\text { Ultrasound of cerebral veins } \\
\text { and sinuses }\end{array}$ & No & Yes & Avoid repetitive CTV or MRV in monitoring COVID-19 patients with CVST ${ }^{33,34}$ \\
\hline ICP surrogates & Yes & Yes & Avoid insertion of EVD in critically ill mechanically ventilated COVID-19 patients ${ }^{44}$ \\
\hline Brain death confirmation & Yes & Yes & Avoid other neuroimaging techniques ${ }^{47,48}$ \\
\hline
\end{tabular}

COVID-19; coronavirus disease 2019, TCD; transcranial Doppler, TCCD; transcranial color-coded duplex, CTA; computed tomography angiography, LVO; large vessel occlusion, CT; computed tomography, RLS; right-to-left-shunt, TEE; transesophageal echocardiography, CTV; computed tomography venography, MRV; magnetic resonance venography, CVST; cerebral venous sinus thrombosis, ICP; intracranial pressure, EVD; external ventricular. 
a recent Cochrane review that assessed the diagnostic accuracy of TCD and transcranial color-coded duplex (TCCD) for detecting stenosis and occlusion of intracranial large arteries in people with acute ischemic stroke, TCD or TCCD when administered by professionals with adequate experience and skills, provide useful diagnostic information for detecting stenosis or occlusion of intracranial vessels with a summary sensitivity of $95 \%$ (95\% confidence interval [CI] $0.83-0.99$ ) and a summary specificity of $95 \%$ (95\% CI $0.95-0.98)$ of all included studies. ${ }^{13}$ Extended applications such as vasomotor reactivity (VMR) testing, emboli monitoring, and right-to-left shunt detection help clinicians ascertain stroke mechanisms at the bedside without transferring unnecessary the patients to radiology or cardiology departments, plan and monitor treatment, and determine prognosis. ${ }^{14}$ By assessing VMR, patients that are at high risk for stroke recurrence in the setting of symptomatic extracranial internal carotid artery stenosis or occlusion can be identified. Thus, microembolic signals (MES) on TCD in these patients could initiate early carotid endarterectomy. Nevertheless, evidence of right-to-left shunts as indicated by TCD could imply paradoxical embolism as a stroke mechanism. ${ }^{11}$

\section{NEUROSONOLOGY APPLICATIONS AND DE- TECTION OF MES}

TCD may provide direct evidence of microemboli circulating in cerebral arteries in real-time. MES can be detected as signals of high intensity and short duration within the Doppler spectrum (Fig. 1). They represent solid or gaseous particles within the blood flow. Although not causing immediate symptoms, these embolic signals are clinically important, as they can identify an embolic mechanism and point to the source of embolism in patients with stroke or transient ischemic attack. TCD represents the gold standard of detection, quantification, and localization of cerebral embolization in real time. ${ }^{11}$

Two studies were identified that evaluated MES detection in COVID-19 patients. ${ }^{15,16}$ Batra et al. ${ }^{15}$ report on six patients with confirmed COVID-19 (four with confirmed ischemic stroke and two with encephalopathy) who underwent TCD monitoring for microemboli detection. MES were identified in three patients, including one who was therapeutically anticoagulated. ${ }^{15}$ In contrast with these findings, TCD could not detect MES in 16 critically ill COVID-19 patients. Nevertheless, it has to be acknowledged that none of these patients had a history of stroke or encephalopathy and the duration of TCD monitoring for emboli detection was very short (15 minutes). ${ }^{16}$

Numerous potential mechanisms for emboli formation have been described in COVID-19. A recent autopsy series provided preliminary evidence of hypercoagulability as well as endotheliitis and endothelial dysfunction with microthrombi observed in multiple organ systems, including the brain. ${ }^{17} \mathrm{Klok}$ et al. ${ }^{18}$ re-

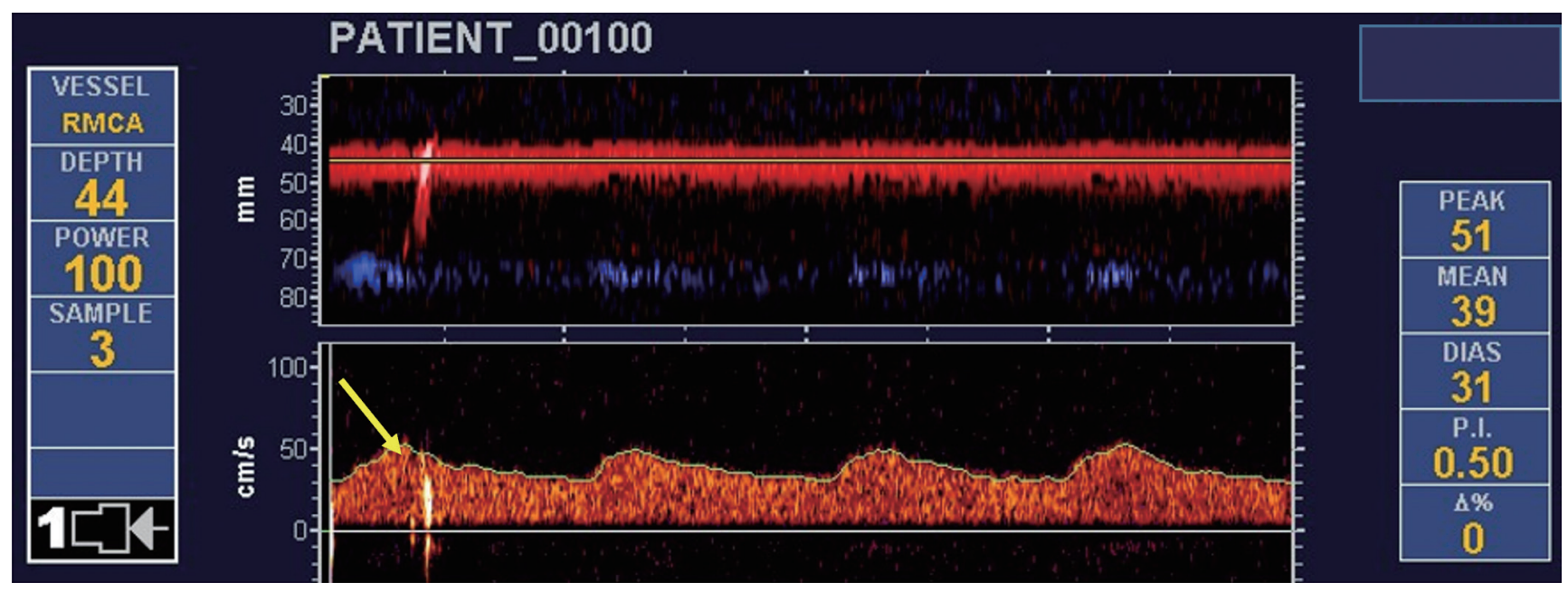

FIG. 1. Transcranial power motion-mode Doppler sonography of the right middle cerebral artery depicting a high-intensity microembolic signal (yellow arrow) in spectral display. 
ported a remarkably high incidence of thrombotic complications in $31 \%$ intensive care units (ICU) patients with COVID-19.

As TCD provides a non-invasive method for evaluating cerebral microemboli in patients with COVID-19, it may be useful in assessing response to treatment in COVID-19 cases with suspected or confirmed disorders of hypercoagulability.

\section{CEREBRAL HEMODYNAMICS ASSESSED BY NEUROSONOLOGY IN PATIENTS WITH COVID-19 DISEASE}

Cerebral autoregulation is mechanistically complex and depends on myogenic, neuronal, endothelial, and metabolic factors. Among numerous methods of estimating cerebrovascular reserve (CVR) TCD presents a non-invasively, easily applicable method. CVR refers to the capacity of the brain to increase cerebral blood volume to maintain a constant regional cerebral blood flow (rCBF) in the face of low cerebral perfusion pressure. ${ }^{19}$

VMR assessed by TCD using vasodilating or constricting stimuli is not a direct measure of autoregulation. ${ }^{20}$ Brain autoregulation maintains $\mathrm{rCBF}$ constant with physiological variations in blood pressure. The changes in $\mathrm{CO}_{2}$ concentration induce a vasomotor response that changes $\mathrm{rCBF}$ in parallel to the velocity changes. Thus, mean flow velocity (MFV) changes on TCD during normo-, hyper-, and hypo-capnia may prove a useful index of VMR and the capacity of smaller cerebral arteries to adapt to various stimuli. ${ }^{19}$

VMR is measured by the breath-holding index (BHI), which is the ratio of the percent MFV increase during hypercapnia over the time (seconds) of breath-holding. ${ }^{21}$ Baseline MFV are obtained during inhalation of room air (rest) followed by a 30 seconds breath-holding effort, followed by a 4 seconds recording of the highest MFV VMR can be quantified by TCD using the BHI:

$\mathrm{BHI}=\left(\left[\mathrm{MFV}_{\max }-\mathrm{MFV}_{\max }\right] \times 100 / \mathrm{MFVbas}\right) / 30$

VMR with the BHI has been studied in 20 COVID-19 patients and 20 healthy volunteers. The authors reported an intriguing finding; they documented an increase in CBF velocity coupled by a decrease in VMR in patients with COVID-19 compared to controls, sug- gesting that this response may be the a result of endothelial dysfunction in the vascular structures of central nervous system. ${ }^{22}$ The working hypothesis for that statement raises form the fact that it has been shown histopathologically that SARS-CoV-2 virus causes endothelial damage. ${ }^{23}$ The vascular endothelium is an active paracrine, endocrine, and autocrine organ that is indispensable for the regulation of vascular tone and the maintenance of vascular homoeostasis. ${ }^{24}$ The decrease of VMR as assessed by TCD may therefore reflect an underlying endothelial dysfunction.

Based on the above mentioned hypothesis another study aimed to assess cerebral VMR with TCD and BHI in 25 patients with mild SARS-CoV-2 infection. ${ }^{25}$ In line with the results of the previous study, the authors found lower BHI values compared to the control group also highlighting the fact that the impairment of cerebral vasoreactivity may be attributed to SARS-CoV-2 virus neurotropicity resulting in impaired endothelial function.

Ziai et al. ${ }^{16}$ observed an unexpected finding regarding cerebral blood flow velocity in COVID-19 patients hospitalized in ICU. They reported relatively low cerebral velocities despite the coexisting low hematocrit levels. ${ }^{16}$ It is known that hematocrit and viscosity are inversely related to cerebral blood flow velocity that increases by $\approx 20 \%$ with a drop in hematocrit from $40 \%$ to $30 \%{ }^{26}$ The authors hypothesized that lower than expected CBF velocities may be explained by a global low flow state due to cardiac systolic dysfunction. This observation deserves further investigation in a larger sample of COVID-19 patients admitted to ICU.

\section{CEREBRAL HEMODYNAMICS ASSESSED BY NEUROSONOLOGY IN HEALTHCARE WORK- ERS}

Bharatendu et al. ${ }^{27}$ have recently investigated the alterations in cerebral hemodynamics in 154 frontline healthcare workers using serial TCD measurements. Previous reports had described significant increases in headache among that group who used PPE comprising of $\mathrm{N}_{95}$ respirator, protective gown and gloves for prolonged hours during the COVID pandemic. ${ }^{28}$ In the study of Bharatendu et al. ${ }^{27}$, the authors evaluated TCD 
parameters including MEFVs and pulsaltility index in middle cerebral artery in parallel with measurements of the end-tidal carbon dioxide $\left(\mathrm{ET}-\mathrm{CO}_{2}\right)$ monitored by a capnometer. $\mathrm{PaCO}_{2}$ is the most important determinant of the vascular tone in intracranial circulation and a rise in partial pressure of carbon dioxide by $1 \mathrm{mmHg}$ proportionately increases CBF by $3-6 \%$. The authors reported that the use of $\mathrm{N}_{95}$ respirator-mask resulted in significant alterations in cerebral hemodynamics. In particular, among participants who developed de novo headaches the use of $\mathrm{N}_{95}$ respirator mask caused significant increase in MCA-MFV and ET- $\mathrm{CO}_{2}$, coupled with reduction in PI. However, these effects were mitigated by the use of additional powered air-purifying respirators (PAPR) as they observed that MFV increased milder in this subgroup of patients while PI remained similar compared to baseline. The investigators concluded that PPE might cause headache to health workers due to the increase in cerebral carbon dioxide causing cerebral vasodilation. This effect was substantially attenuated by the use of additional PAPR. Therefore, the use of PAPR together with the N95 mask was recommended for healthcare-workers with longer duties in the hospital wards.

\section{NEUROSONOLOGY APPLICATIONS IN THE DIAGNOSIS AND MONITORING OF CERE- BRAL SINUS OR VENOUS THROMBOSIS IN COVID-19 PATIENTS}

Cerebral venous sinus thrombosis (CVST) is a wellknown complication in COVID-19 patients. A multinational study including 432 patients COVID-19 with stroke identified 18 patients (4.2\%) with cerebral venous or sinus thrombosis. ${ }^{29}$

Regarding the imaging detection of CVST, it mainly relies on computed tomography venography (CTV), magnetic resonance venography (MRV), or digital subtraction angiography. However, the aforementioned imaging techniques cannot reflect the hemodynamic changes in deep cerebral veins, their use in COVID-19 patients is often problematic and their use for repeated monitoring during a short time is not easily applicable. ${ }^{30}$

Venous ultrasound techniques have been developed in the mid-1990 with the aim to serve as a noninvasive, easy to use and cost-effective screening method for CVST. ${ }^{31}$ With the development of high resolution ultrasound systems and the advent of the second-generation ultrasound contrast agents, ultrasound diagnosis of intracranial venous diseases appears to have a satisfactory sensitivity among experience neurosonologists. ${ }^{32}$

TCCD enables measurements of cerebral blood flow velocities in deep cerebral veins, such as the deep middle cerebral veins, basal vein of Rosenthal, vein of Galen but also in straight sinus, transverse sinus, superior sagittal sinus, sphenoparietal sinus, superior petrosal sinus and inferior petrosal sinus. ${ }^{31}$ Moreover, TCCD can perform serial velocity measurements in venous collaterals pathways in patients with acute CVST. ${ }^{33,34}$ The latter is important because it has been shown that there is an clear relationship between initially high venous flow velocities in patients with CVST and the severity of disease..$^{31}$ Furthermore, in the prospective study of Stolz et al..$^{34}$ including 26 patients with acute CVST, an initially normal venous ultrasound examination or normalization within 90 days were significantly associated with an excellent functional outcome (modified Rankin Scale score o or 1). This highlights the importance of hemodynamic factors for the long-term outcome of CVST which cannot be assessed by current routine CTV and MRV.

TCCD may therefore serve as a non-invasive, cost-effective examination technique in the diagnosis and mainly in the follow-up of patients with CVST providing prognostic information on venous hemodynamics and collateral pathways. So far, there are no studies with application of TCCD in COVID-19 patients suffering from CVST but in the setting of COVID-19 where MRI is not readily available, the diagnostic role of TCCD emerges with increasing clinical relevance.

\section{NEUROSONOLOGY APPLICATIONS IN CRITI- CALLY ILL COVID-19 PATIENTS}

COVID-19 is associated with severe disease requiring intensive care in approximately $5 \%$ of proven infections. ${ }^{35}$ Several neurological complications which may lead patients to ICU are associated with intracranial hypertension, which is a potentially fatal condition..$^{6}$ ICP 
monitoring becomes fundamental in order to facilitate prompt diagnosis and appropriate therapeutic decision-making. The gold standard for continuous ICP monitoring remains the invasive measurement through insertion of a catheter within the brain ventricles connected to an external pressure transducer. ${ }^{37}$ However, this method may be not always available especially in times of limited resources as in COVID-19 pandemic. Additionally invasive ICP monitoring may increase the risk of serious complications, such as infections or intracranial hemorrhage. ${ }^{38,39}$ Noninvasive neuromonitoring systems are increasingly used in neurointensive care settings for patients with primary cerebral damage but also in critically ill patients as useful tools to detect neurological complications causing intracranial hypertension. ${ }^{40}$ In particular, the pulsatility index evaluation by TCD and optic nerve sheath diameter (ONSD) measurement by TCCD can be applied safely at the patient's bedside to assess cerebral hemodynamics as well as to monitor CPP and ICP noninvasively. ${ }^{41}$ It should be mentioned that none of these methods have been established in daily clinical practice and a better understanding of their diagnostic accuracy is still necessary.

TCD shows spectral waveforms that represent the depth, direction, and intensity of the blood flow through the intracranial vasculature. Although TCD does not measure blood flow directly, the parameters

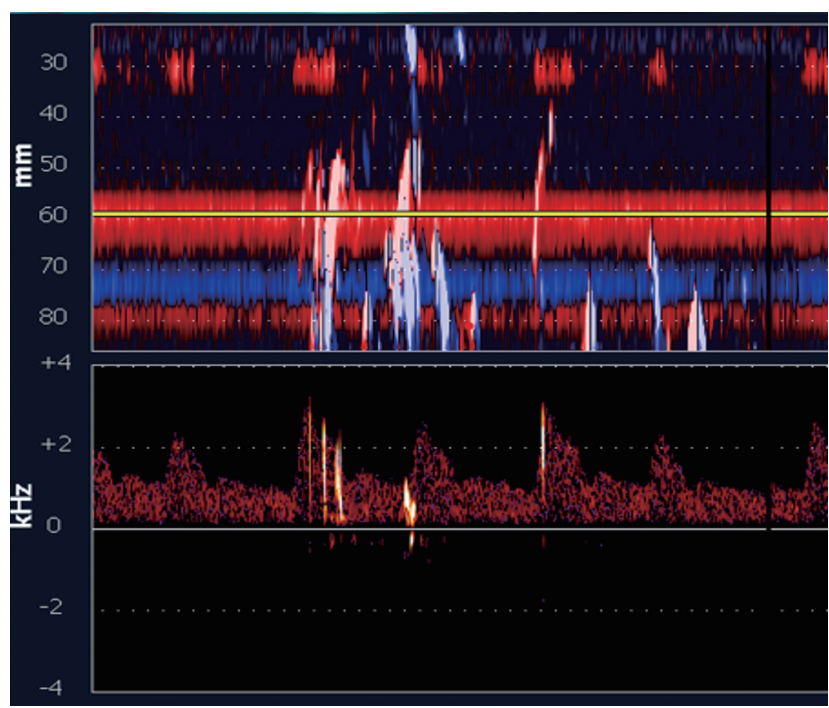

FIG. 2. Transcranial power motion-mode Doppler demonstrating detection of multiple microembolic signals after injection of agitated normal saline indicating right-to left shunt. that it calculates correlate with $\mathrm{CBF} \cdot{ }^{41}$

ICP can be also indirectly evaluated using ONSD measurements. ${ }^{42}$ Brain CT studies have showed a correlation between high ICP and increasing ONSD. ${ }^{43}$ In order to measure ONSD a $7.5-\mathrm{MHz}$ to $10-\mathrm{MHz}$ linear array transducer is placed on the patient's closed eye after applying ultrasonography gel. The optic nerve sheath is marked $3 \mathrm{~mm}$ behind the posterior aspect of the eye and measurement of the transverse diameter of the optic nerve sheath is obtained at this position. If it exceeds $5 \mathrm{~mm}$, this indicates increased ICP. ${ }^{41}$

A recent study conducted by Battaglini et al..$^{44}$ aimed to assess changes in cerebral hemodynamics, their effects on outcome, and their role as potential predictors of neurological complications in 53 mechanically ventilated COVID-19 patients by applying TCD and ONSD measurements. One of the main findings of the study was that increased ICP as estimated by ONSD was common in these patients and was also associated with longer ICU length of stay. This confirms that, in COVID-19 patients, noninvasive ICP monitoring may be essential for the early detection of patients who are at risk of longer hospitalization in the ICU with subsequent complications and difficult recovery.

Another single-center prospective study with 50 COVID-19 critically ill and mechanically ventilated patients showed that early alteration of cerebral hemodynamics (as evaluated by TCD) in combination with assessment of intracranial compliance (as evaluated by cranial deformation) were associated with increased

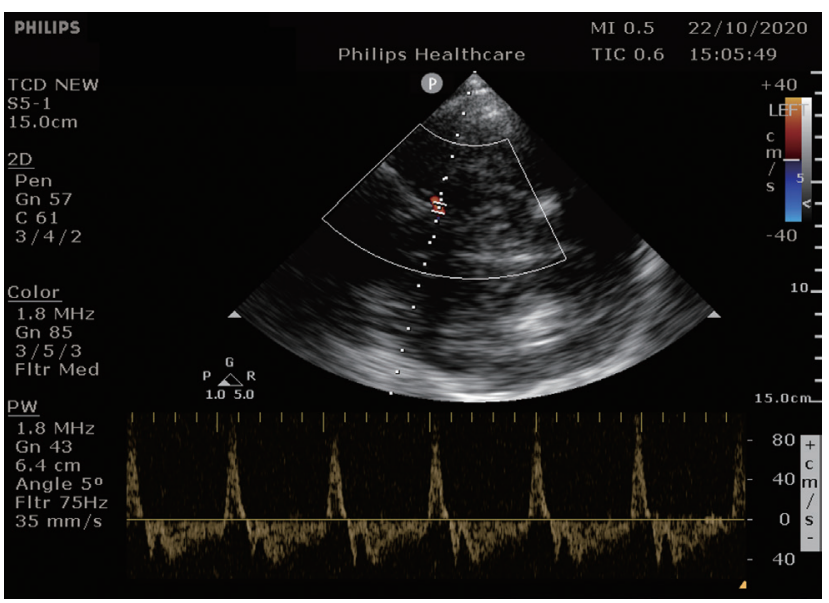

FIG. 3. Transcranial color-coded, sonography showing reverberating flow in $M_{1}$ middle cerebral artery indicative of circulatory arrest. 
severity of COVID-19, including death or dependency from mechanical ventilation. ${ }^{45}$

All the above-mentioned findings advocate for larger investigations on the role of neuro-monitoring in order to detect earlier all relevant ultrasound parameters that are important for the outcome of critically ill COVID-19 patients.

\section{NEUROSONOLOGY APPLICATIONS IN DE- TECTING PULMONARY VASCULAR DILATATION IN PATIENTS WITH COVID-19 PNEUMONIA}

Reynolds et al. ${ }^{46}$ aimed to provide insights into the pathophysiology of hypoxemic respiratory disorders in COVID-19 by applying TCD with intravenous injection of agitated normal saline in 18 mechanically ventilated patients with severe COVID-19 pneumonia. They performed TCD-bubble studies in order to detect potential right-to left shunt (Fig. 2) and had three interesting findings: 1) $83 \%$ of patients had detectable MES over 20 seconds of monitoring after injection of agitated normal saline, 2) $\mathrm{PaO}_{2}: \mathrm{FIO}_{2}$ ratio was inversely correlated with the number of MES and 3) the number of MES was inversely correlated to lung compliance. Based on these findings, the authors suggest that pulmonary vasodilatations may explain the disproportionate hypoxemia in some patients with COVID-19 pneumonia.

\section{NEUROSONOLOGY APPLICATIONS IN BRAIN DEATH DIAGNOSIS IN COVID-19}

Brain death remains a clinical diagnosis requiring a variety of clinical, laboratory, and respiratory criteria. ${ }^{43}$ Many factors like sedation, drug intoxication, hypothermia, neuromuscular paralysis, severe hemodynamic instability, significant electrolyte imbalance may preclude the satisfaction of the clinical criteria. In such situations, ancillary tests play a vital role to confirm brain death and to decrease futile observation time. ${ }^{47}$ TCD provides a useful ancillary test for cerebral circulatory arrest confirmation because it is safe, noninvasive, and it can be easily performed at the bedside. On TCD, cerebral circulatory arrest is indicated by flow patterns with increased pulsatility, followed by reduc- tion, elimination, and reversal of diastolic flow. Finally, a reverberating flow pattern emerges, and, at that point, TCD can confirm complete cerebral circulatory arrest (Fig. 3). Absence of flow can only be used as a marker for cerebral circulatory arrest if an acoustic signal that had previously been obtained in the same patient is now absent. $^{48}$

For mechanically ventilated COVID-19 patients in whom brain death is clinically assumed, Kapoor et al. ${ }^{49}$ suggest that TCD represents an ancillary test method for confirmation of cerebral circulatory arrest with lower infection risk for the healthcare providers, allowing to avoid a potential virus transmission compared to apnea test. In addition, CT angiography, which is currently in wide clinical use $\mathrm{e}^{50}$ is also more time consuming and requires the involvement of more staff which in COVID-19 setting should preferably be avoided.

\section{CONCLUSIONS}

COVID-19 pandemic has forced all affected healthcare systems to re-organize their diagnostic pathways in order to provide optimal care and minimize the risk of transmission. In this context neurosonology, which represents a non-invasive, easily applicable at the bedside and cost-effective diagnostic tool serves may provide invaluable and complementary to other imaging modalities diagnostic information. In the setting of acute stroke it may help determine the reperfusion treatment. Furthermore, it reliably contributes to diagnostic work-up of stroke patients. Thus, neurosonologists should be familiar with extended neurosonology applications and the use of TCD/TCCD in the neurointensive care setting.

\section{Ethics Statement}

The article is original and has not been published elsewhere in any form or language.

\section{Availability of Data and Material}

The data that support the findings of this study are available in the text.

\section{Acknowledgments}

None. 


\section{Sources of Funding}

None.

\section{Conflicts of Interest}

No potential conflicts of interest relevant to this article was reported.

\section{REFERENCES}

1. The Johns Hopkins University and Medicine. COVID-19 dashboard by the center for systems science and engineering (CSSE) at Johns Hopkins University (JHU). [Internet]. Coronavirus Resource Center. 2021 [cited 2021 Aug 23]. Available from https://coronavirus.jhu.edu/map.html.

2. Robba C, Battaglini D, Pelosi P, Rocco PRM. Multiple organ dysfunction in SARS-CoV-2: MODS-CoV-2. Expert Rev Respir Med. 2020;14:865-868.

3. Lai CC, Ko WC, Lee PI, Jean SS, Hsueh PR. Extra-respiratory manifestations of COVID-19. Int J Antimicrob Agents. 2020;56:106024.

4. Gavriatopoulou M, Korompoki E, Fotiou D, Ntanasis-Stathopoulos I, Psaltopoulou T, Kastritis E, et al. Organ-specific manifestations of COVID-19 infection. Clin Exp Med. 2020;20:493-506.

5. Tsivgoulis G, Palaiodimou L, Katsanos AH, Caso V, Köhrmann M, Molina C, et al. Neurological manifestations and implications of COVID-19 pandemic. Ther Adv Neurol Disord. 2020 Jun 9. doi: 10.1177/1756286420932036 [Epub ahead of print].

6. Tsivgoulis G, Palaiodimou L, Zand R, Lioutas VA, Krogias C, Katsanos AH, et al. COVID-19 and cerebrovascular diseases: a comprehensive overview. Ther Adv Neurol Disord. 2020 Dec 8. doi: 10.1177/1756286420978004 [Epub ahead of print].

7. Katsanos AH, Palaiodimou L, Zand R, Yaghi S, Kamel H, Navi BB, et al. The impact of SARS-CoV-2 on stroke epidemiology and care: a meta-analysis. Ann Neurol. 2021;89:380388.

8. Zini A, Romoli M, Gentile M, Migliaccio L, Picoco C, Dell'Arciprete $\mathrm{O}$, et al. The stroke mothership model survived during COVID-19 era: an observational single-center study in Emilia-Romagna, Italy. Neurol Sci. 2020;41:3395-3399.

9. Baracchini C, Pieroni A, Kneihsl M, Azevedo E, Diomedi M, Pascazio L, et al. Practice recommendations for neurovascular ultrasound investigations of acute stroke patients in the setting of the COVID-19 pandemic: an expert con- sensus from the European Society of Neurosonology and Cerebral Hemodynamics. Eur J Neurol. 2020;27:1776-1780.

10. Chernyshev OY, Garami Z, Calleja S, Song J, Campbell MS, Noser EA, et al. Yield and accuracy of urgent combined carotid/transcranial ultrasound testing in acute cerebral ischemia. Stroke. 2005;36:32-37.

11. Tsivgoulis G, Alexandrov AV. Ultrasound in neurology. Continuum (Minneap Minn). 2016;22:1655-1677.

12. Tsivgoulis G, Ribo M, Rubiera M, Vasdekis SN, Barlinn K, Athanasiadis D, et al. Real-time validation of transcranial Doppler criteria in assessing recanalization during intra-arterial procedures for acute ischemic stroke: an international, multicenter study. Stroke. 2013;44:394-400.

13. Mattioni A, Cenciarelli S, Eusebi P, Brazzelli M, Mazzoli T, Del Sette M, et al. Transcranial Doppler sonography for detecting stenosis or occlusion of intracranial arteries in people with acute ischaemic stroke. Cochrane Database Syst Rev. 2020;2:CDo10722.

14. Tsivgoulis G, Alexandrov AV, Sloan MA. Advances in transcranial Doppler ultrasonography. Curr Neurol Neurosci Rep. 2009;9:46-54

15. Batra A, Clark JR, LaHaye K, Shlobin NA, Hoffman SC, Orban ZS, et al. Transcranial Doppler ultrasound evidence of active cerebral embolization in COVID-19. J Stroke Cerebrovasc Dis. 2021;30:105542.

16. Ziai WC, Cho SM, Johansen MC, Ergin B, Bahouth MN. Transcranial Doppler in acute COVID-19 infection: unexpected associations. Stroke. 2021;52:2422-2426.

17. Bryce C, Grimes Z, Pujadas E, Ahuja S, Beasley MB, Albrecht $\mathrm{R}$, et al. Pathophysiology of SARS-CoV-2: targeting of endothelial cells renders a complex disease with thrombotic microangiopathy and aberrant immune response. The Mount Sinai COVID-19 autopsy experience. medRxiv. 2020 May 22. doi: 10.1101/2020.05.18.20099960 [preprint].

18. Klok FA, Kruip MJHA, van der Meer NJM, Arbous MS, Gommers DAMPJ, Kant KM, et al. Incidence of thrombotic complications in critically ill ICU patients with COVID-19. Thromb Res. 2020;191:145-147.

19. Tsivgoulis G, Alexandrov AV. Cerebral hemodynamics in acute stroke: pathophysiology and clinical implications. J Vasc Interv Neurol. 2008;1:65-69.

20. Sharma VK, Tsivgoulis G, Lao AY, Alexandrov AV. Role of transcranial Doppler ultrasonography in evaluation of patients with cerebrovascular disease. Curr Neurol Neurosci Rep. 2007;7:8-20.

21. Markus HS, Harrison MJ. Estimation of cerebrovascular 
reactivity using transcranial Doppler, including the use of breath-holding as the vasodilatory stimulus. Stroke. 1992;23:668-673.

22. Sonkaya AR, Öztrk B, Karadaş Ö. Cerebral hemodynamic alterations in patients with COVID-19. Turk J Med Sci. 2021;51:435-439.

23. Varga Z, Flammer AJ, Steiger P, Haberecker M, Andermatt R, Zinkernagel AS, et al. Endothelial cell infection and endotheliitis in COVID-19. Lancet. 2020;395:1417-1418.

24. Flammer AJ, Anderson T, Celermajer DS, Creager MA, Deanfield J, Ganz P, et al. The assessment of endothelial function: from research into clinical practice. Circulation. 2012;126:753-767.

25. Marcic M, Marcic L, Marcic B, Capkun V, Vukojevic K. Cerebral vasoreactivity evaluated by transcranial color Doppler and breath-holding test in patients after SARS-CoV-2 infection. J Pers Med. 2021;11:379.

26. Gaehtgens P, Marx P. Hemorheological aspects of the pathophysiology of cerebral ischemia. J Cereb Blood Flow Metab. 1987;7:259-265.

27. Bharatendu C, Ong JJY, Goh Y, Tan BYQ, Chan ACY, Tang JZY, et al. Powered air purifying respirator (PAPR) restores the N95 face mask induced cerebral hemodynamic alterations among Healthcare Workers during COVID-19 outbreak. J Neurol Sci. 2020;417:117078.

28. Ong JJY, Bharatendu C, Goh Y, Tang JZY, Sooi KWX, Tan YL, et al. Headaches associated with personal protective equipment - a cross-sectional study among frontline Healthcare Workers during COVID-19. Headache. 2020;60:864-877.

29. Shahjouei S, Tsivgoulis G, Farahmand G, Koza E, Mowla A, Vafaei Sadr A, et al. SARS-CoV-2 and stroke characteristics: a report from the multinational COVID-19 stroke study group. Stroke. 2021;52:e117-e130.

30. Weimar C, Masuhr F, Hajjar K. Diagnosis and treatment of cerebral venous thrombosis. Expert Rev Cardiovasc Ther. 2012;10:1545-1553.

31. Stolz EP. Role of ultrasound in diagnosis and management of cerebral vein and sinus thrombosis. Front Neurol Neurosci. 2008;23:112-121.

32. Zhu X, Liu M, Gong X, Jin Z, Wang F, Wei S, et al. Transcranial color-coded sonography for the detection of cerebral veins and sinuses and diagnosis of cerebral venous sinus thrombosis. Ultrasound Med Biol. 2019;45:2649-2657.

33. Valdueza JM, Hoffmann O, Weih M, Mehraein S, Einhäupl KM. Monitoring of venous hemodynamics in patients with cerebral venous thrombosis by transcranial Doppler ultra- sound. Arch Neurol. 1999;56:229-234.

34. Stolz E, Gerriets T, Bödeker RH, Hügens-Penzel M, Kaps M. Intracranial venous hemodynamics is a factor related to a favorable outcome in cerebral venous thrombosis. Stroke. 2002;33:1645-1650.

35. Wu Z, McGoogan JM. Characteristics of and important lessons from the coronavirus disease 2019 (COVID-19) outbreak in China: summary of a report of 72314 cases from the Chinese Center for Disease Control and Prevention. JAMA. 2020;323:1239-1242.

36. Güiza F, Depreitere B, Piper I, Citerio G, Chambers I, Jones $\mathrm{PA}$, et al. Visualizing the pressure and time burden of intracranial hypertension in adult and paediatric traumatic brain injury. Intensive Care Med. 2015;41:1067-1076.

37. Bhatia A, Gupta AK. Neuromonitoring in the intensive care unit. I. Intracranial pressure and cerebral blood flow monitoring. Intensive Care Med. 2007;33:1263-1271.

38. Holloway KL, Barnes T, Choi S, Bullock R, Marshall LF, Eisenberg HM, et al. Ventriculostomy infections: the effect of monitoring duration and catheter exchange in 584 patients. J Neurosurg. 1996;85:419-424.

39. Binz DD, Toussaint LG 3rd, Friedman JA. Hemorrhagic complications of ventriculostomy placement: a meta-analysis. Neurocrit Care. 2009;10:253-256.

40. Fernando SM, Tran A, Cheng W, Rochwerg B, Taljaard M, Kyeremanteng K, et al. Diagnosis of elevated intracranial pressure in critically ill adults: systematic review and meta-analysis. BMJ. 2019;366:14225.

41. Hakimi R, Alexandrov AV, Garami Z. Neuro-ultrasonography. Neurol Clin. 2020;38:215-229.

42. Shin SS, Huisman TAGM, Hwang M. Ultrasound imaging for traumatic brain injury. J Ultrasound Med. 2018;37:18571867.

43. Masquère P, Bonneville F, Geeraerts T. Optic nerve sheath diameter on initial brain CT, raised intracranial pressure and mortality after severe TBI: an interesting link needing confirmation. Crit Care. 2013;17:151.

44. Battaglini D, Santori G, Chandraptham K, Iannuzzi F, Bastianello M, Tarantino F, et al. Neurological complications and noninvasive multimodal neuromonitoring in critically ill mechanically ventilated COVID-19 patients. Front Neurol. 2020;11:602114.

45. Brasil S, Taccone FS, Wayhs SY, Tomazini BM, Annoni F, Fonseca $S$, et al. Cerebral hemodynamics and intracranial compliance impairment in critically ill COVID-19 patients: a pilot study. Brain Sci. 2021;11:874. 
46. Reynolds AS, Lee AG, Renz J, DeSantis K, Liang J, Powell CA, et al. Pulmonary vascular dilatation detected by automated transcranial Doppler in COVID-19 pneumonia. Am J Respir Crit Care Med. 2020;202:1037-1039.

47. Chang JJ, Tsivgoulis G, Katsanos AH, Malkoff MD, Alexandrov AV. Diagnostic accuracy of transcranial Doppler for brain death confirmation: systematic review and meta-analysis. AJNR Am J Neuroradiol. 2016;37:408-414.

48. Kramer AH. Ancillary testing in brain death. Semin Neurol.
2015;35:125-138.

49. Kapoor I, Prabhakar H, Mahajan C. COVID-19 and diagnosing brain death: an ambiguity. Turk J Anaesthesiol Reanim. $2020 ; 48: 436$.

50. Taylor T, Dineen RA, Gardiner DC, Buss CH, Howatson A, Pace NL. Computed tomography (CT) angiography for confirmation of the clinical diagnosis of brain death. Cochrane Database Syst Rev. 2014;2014:CDoo9694. 